\title{
Port-Site Resection in Surgical Management of Incidental Gallbladder Cancer: A Still Unresolved Question
}

\author{
Quanda Liu, MD ${ }^{1}$, Naixin $\mathrm{Wu}, \mathrm{MD}^{2}$, and Ningxin $\mathrm{Zhou}, \mathrm{MD}^{1}$ \\ ${ }^{1}$ Department of Hepatobiliary Surgery, Second Artillery General Hospital PLA, Beijing, China; ${ }^{2}$ Shanghai Jiao Tong \\ University, Shanghai, China
}

\section{TO THE EDITORS:}

We read with great interest the article by Dr. Maker et al., ${ }^{1}$ in which patients undergoing definitive resection for incidental gallbladder carcinoma (GBCA) after laparoscopic cholecystectomy (LC) were divided into two groups: laparoscopic port site resected (group 1, $n=69$ ) and laparoscopic port site not resected (group 2, $n=44$ ). The authors reported that the incidence of port-site metastases (PSMs) was as high as $19 \%$, and median survival of patients with T2/T3 tumors without PSMs was 42 months compared with 17 months in patients with PSMs $(P=0.005)$, while excision of port sites did not affect overall survival or disease recurrence, so that port-site resection should not be considered mandatory during definitive surgical treatment of incidental GBCA.

However, the study has two major defects making the intergroup clinicopathological features incomparable, thus probably demolishing the validity of the conclusions: (a) 12 patients $(17 \%)$ underwent $\mathrm{R} 2$ resection in group 1 , but only 1 patient $(2 \%)$ in group $1(P=0.002)$; (b) 40 patients $(58 \%)$ received chemotherapy in group 1 , whereas 5 patients $(11 \%)$ received chemotherapy in group $2(P=$ $0.000)$. We know that survival was improved in patients with cholangiocarcinoma who underwent R0 and R1 resection compared with those undergoing $\mathrm{R} 2$ resection, ${ }^{2}$ so the higher proportion of R2 resection inevitably impaired survival in group 1. Recent studies have demonstrated that chemotherapy can also significantly prolong survival for patients with biliary tract cancers, ${ }^{3}$ so

\section{(C) Society of Surgical Oncology 2017}

First Received: 29 October 2017;

Published Online: 20 November 2017

Q. Liu, MD

e-mail: liuquanda@sina.com a low ratio of chemotherapy of patients in group 2 certainly decreased survival. The above-mentioned intergroup biases blurred the survival outcomes.

There are some other flaws: (i) The absence of uniform diagnostic criteria of PSM(s), namely pathologic diagnosis in group 1 versus clinical diagnosis by symptomatic and radiologic evidence in group 2, resulted in an anamorphic incidence of PSM. In general, all port-site micro-metastases and some small macro-metastases could not be detected clinically; (ii) 16 out of 44 patients (36\%) in group 2 underwent conversion of laparoscopic to open surgery, ranging from explorative laparoscopy to complete LC, which are incomparable with the complete laparoscopic procedures in group 1. The more laparoscopic procedures that patients in group 1 received, the higher the risk of PSM occurring; (iii) the interval time from LC to port-site resection was not mentioned.

We expect that a redesigned study excluding aforesaid biases could shed light on the controversial subject of whether port sites should be resected after LC for incidental GBCA.

DISCLOSURE None.

\section{REFERENCES}

1. Maker AV, Butte JM, Oxenberg J, et al. Is port site resection necessary in the surgical management of gallbladder cancer? Ann Surg Oncol. 2012;19:409-417. https://doi.org/10.1245/s10434011-1850-9. PMID: 21698501.

2. Schiffman SC, Reuter NP, McMasters KM, et al. Overall survival peri-hilar cholangiocarcinoma: R1 resection with curative intent compared to primary endoscopic therapy. J Surg Oncol. 2012;105:91-96.

3. Romiti A, D'Antonio C, Zullo A, et al. Chemotherapy for the biliary tract cancers: moving toward improved survival time. $J$ Gastrointest Cancer. 2012;43:396-404. 